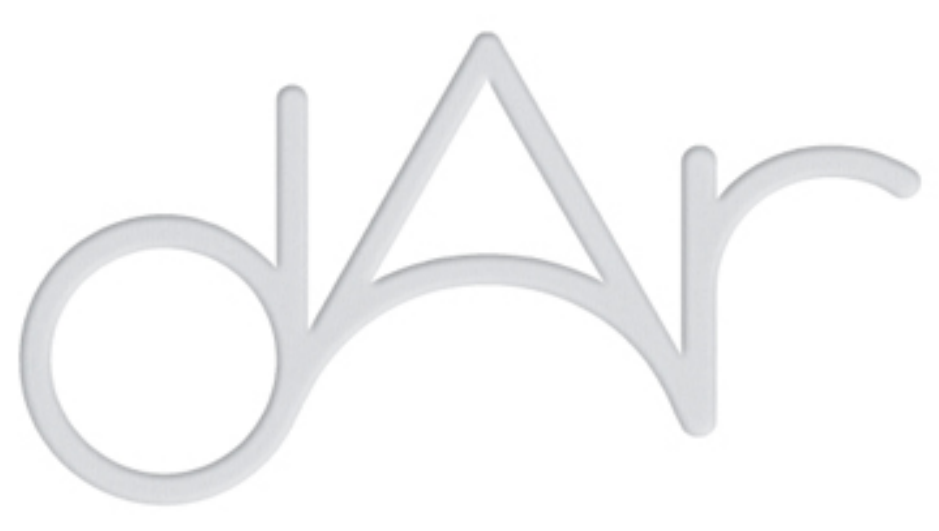

Quels impacts des festivites sur la durabilite du patrimoine archeologique: cas de la Qal'a des Benni Hammad em Algerie

\begin{tabular}{|c|c|}
\hline Autor(es): & $\begin{array}{l}\text { Hynda, Boutabba; Mohamed, Mili; Samir-Djemoui, Boutabba; Abderazak, } \\
\text { Mesaad }\end{array}$ \\
\hline Publicado por: & Imprensa da Universidade de Coimbra \\
\hline $\begin{array}{l}\text { URL } \\
\text { persistente: }\end{array}$ & URI:http://hdl.handle.net/10316.2/44438 \\
\hline DOI: & DOI:https://doi.org/10.14195/2182-844X_5_17 \\
\hline Accessed : & 26-Apr-2023 14:39:37 \\
\hline
\end{tabular}

A navegação consulta e descarregamento dos títulos inseridos nas Bibliotecas Digitais UC Digitalis, UC Pombalina e UC Impactum, pressupõem a aceitação plena e sem reservas dos Termos e Condições de Uso destas Bibliotecas Digitais, disponíveis em https://digitalis.uc.pt/pt-pt/termos.

Conforme exposto nos referidos Termos e Condições de Uso, o descarregamento de títulos de acesso restrito requer uma licença válida de autorização devendo o utilizador aceder ao(s) documento(s) a partir de um endereço de IP da instituição detentora da supramencionada licença.

Ao utilizador é apenas permitido o descarregamento para uso pessoal, pelo que o emprego do(s) título(s) descarregado(s) para outro fim, designadamente comercial, carece de autorização do respetivo autor ou editor da obra.

Na medida em que todas as obras da UC Digitalis se encontram protegidas pelo Código do Direito de Autor e Direitos Conexos e demais legislação aplicável, toda a cópia, parcial ou total, deste documento, nos casos em que é legalmente admitida, deverá conter ou fazer-se acompanhar por este aviso.

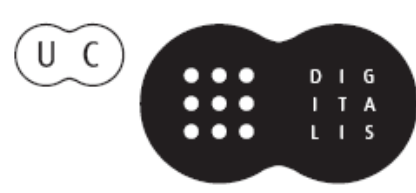



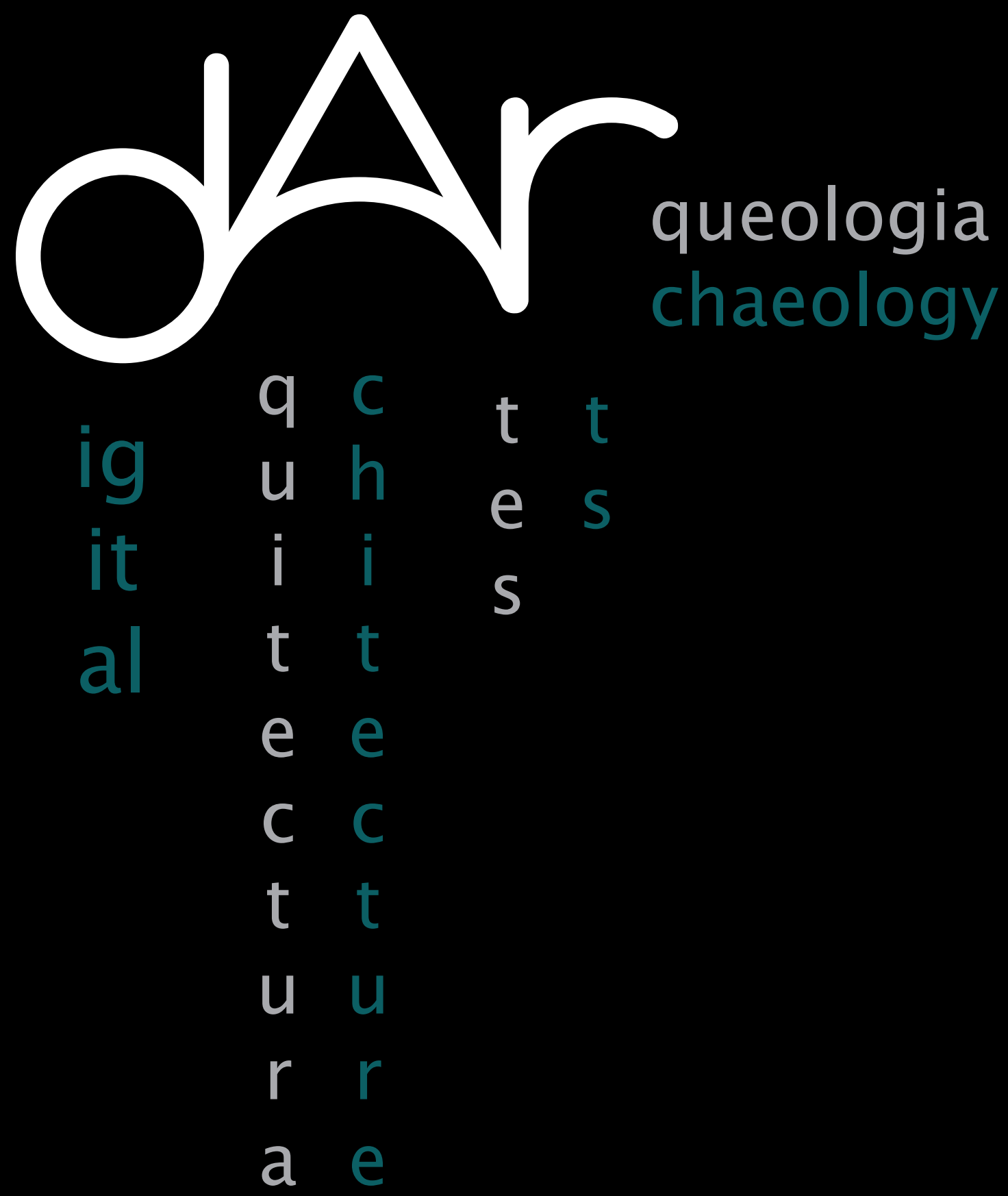

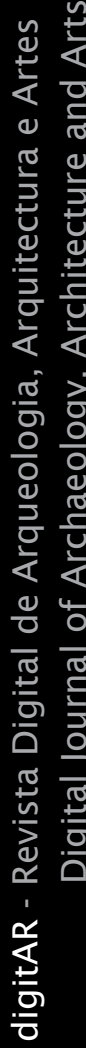




\title{
Quels Impacts des Festivites sur la durabilite du Patrimoine Archeologique: cas de la Qal'a des Benni Hammad em Algerie
}

\author{
Boutabba Hynda ${ }^{1}$, Mili Mohamed ${ }^{2}$, Boutabba Samir-Djemoui ${ }^{3}$ \& Mesaad \\ Abderazak $^{4}$ \\ ${ }^{1}$ Institut de gestion des techniques urbaines, Université de M'sila/ Algérie. \\ ${ }^{2}$ Institut de gestion des techniques urbaines, Université de M'sila/ Algérie. \\ ${ }^{3}$ Département d'architecture, Université de Biskra/Algérie. \\ 4Secrétaire général de l'ordre des architectes Msila/Algérie.
}

\section{Resume}

De par sa position stratégique au carrefour de l'Afrique et de la Méditerranée, l'Algérie, située au centre du Maghreb, a vu de nombreuses civilisations se succéder sur son territoire. Chacune d'elle a laissé un héritage patrimonial ayant une portée mémorielle et symbolique. Classée en 1980, la Qal'a ou citadelle des Benni Hammad est le plus important site archéologique de la dynastie sanhadjienne des Hammadides, née de la scission territoriale du royaume ziride héritier de l'empire fatimide au Maghreb. Situé dans la localité de Béchara, l'une des plus pauvres de la région du Hodna, Ce site archéologique a été choisi comme un levier du développement local, et ce, par l'entremise de sa valorisation et le renforcement de son attractivité touristique. Cependant, le concept de préservation qui est attaché à la définition du patrimoine mondial de l'humanité fait mauvais ménage avec les pratiques actuelles du tourisme qui sont basées sur la notion de flux de personnes. Si le tourisme festif commence à donner un souffle économique et social à la localité de Béchara, le comportement touristique envers les éléments archéologiques de la citadelle Hamadide se fait dans un rythme et des parcours qui ne sont pas forcément positifs pour le site. Le présent papier tente de mettre en exergue les différents impacts de «la fête du printemps» sur le patrimoine de la Qal'a.

Mots-clés: Patrimoine archéologique, tourisme festif, la Qal'a, Algérie. 


\section{Introduction}

La conservation, considérée comme la protection, la préservation, la restauration et présentation des vestiges monumentaux de cultures et civilisations anciennes, avait jouit, dans le monde, depuis le début du siècle dernier d'un intérêt remarquable (Gravari-Barbas \& Jacquot, 2008, 2010 ; Lebaal \& Zérouala, 1997 : 281-289). Depuis le début des années 1990, une autre facette de la conservation du patrimoine a vu le jour, celle de son apport économique en tant qu' offre touristique (Harrison \& Hitchcock, 2005 ; Velle \& Claverie, 2014). En effet, le tourisme et le patrimoine font bon ménage symbiotique, puisque pour le premier c'est l'occasion d'enrichir son offre et pour le deuxième, c'est l'occasion de renforcer sa durabilité par des actions de valorisation (Lazzarotti, 2003 : 98 ; Dailly, Torrente \& Barthes, 2009 :1). À l'instar des autres nations, l'Algérie a placé le patrimoine au centre de ses préoccupations. Elle a établi, depuis1998, une politique du patrimoine fondée sur des actions concrètes, en ratifiant, d'une part, les termes de la charte de Venise ${ }^{1}$ où la notion de monument historique a été largement étayée. D'autre part, en adoptant les résolutions de l'UNESCO afin d'attribuer aux monuments et sites classés la valeur et la place qu'ils méritent (BenseddikSouki \& Chaabi, 2012 :7). Ainsi après l'ordonnance n67-281 du 20 Décembre 1967 complétée par la loi 98-04 du 15 juin 1998 qui étaient, en général dédiées à la préservation du patrimoine, avait été promulguée la loi spécifique 31.01 du 17.02.2003 qui visait l'insertion de l'image de l'Algérie dans le marché international du tourisme, à travers la diversification de l'offre touristique, la préservation de l'environnement et la valorisation du potentiel culturel et historique. C'est dans cette visée qu'a été pensé le développement local de Béchara, à travers la remise en valeur des potentialités touristiques de la Qal'a des Beni Hammad et les festivités du printemps qui se voulaient un atout sûr afin de propulser le potentiel culturel existant dans la région d'une part et de ramener une plus value fondée sur un développement durable de la commune ${ }^{2}$ de Maadid et sa région. Cependant si ces festivités sont perçues positivement sur l'économie de la localité de Béchara, leurs impacts sur

\footnotetext{
1187 des 191 pays membres des Nations Unies, soit 97\% ont ratifié la convention du patrimoine

2 En Algérie, la collectivité territoriale de base est appelée commune. Disposant de la personnalité morale, elle est dotée de ses propres organes, délibératif et exécutif.
} 
la citadelle sont négativement dénoncés. Ces dégradations du patrimoine archéologique proviennent de la faiblesse de la législation algérienne mais également du manque d'éducation et de sensibilisation, et des touristes, et de la population locale en vers cet héritage.

Pour l'aboutissement du déroulement de cette étude, l'enquête s'est déroulée en deux temps

Dans un premier temps : Il sera question de présenter le site, son histoire, ses vestiges architecturaux ainsi que l'état actuel de sa conservation

Dans un second temps : sera présenté le festival, ses approches conceptuelles et historiques. Sera abordé, par la suite, le festival du printemps de la Qal'a ; ses différents impacts sur les localités de Béchara- Maadid ainsi que sur le site archéologique.

\section{Methodologie d'approche}

Les catégories d'analyse liées au tourisme ont été étudiées par divers auteurs classiques en sociologie urbaine que tels que Max Weber, Georg Simmel, Louis Wirth ou Robert Ezra Park. L'enjeu économique du tourisme a fait l'objet de plusieurs travaux (Pearce, 1987 ; Cazes \& Potier, 1996 ; Judd \& Fainstein, 1999). Dans ce sens, la perspective économico-spatiale a prédominé (Law, 1993 ; Page, 1995) Ainsi, la littérature scientifique sur le sujet est assez conséquente. Cependant étant donné le manque flagrant en matière d'information sur la présente étude, cette dernière s'est basée sur une approche inductive et interprétative influencée par le paradigme constructiviste (Roy, 2009: 214 ; Blais \& Martineau, 2006 :4). Pour analyser les impacts et retombées économiques de la fête du printemps sur la commune de Béchara et la daira de Maadid, la méthodologie utilisée s'est s'inspirée de la triangulation des données (Decrop, 2004 : 162). Par l'utilisation d'enquête directe en face-à-face auprès de 100 habitants (50 de la commune de Béchara et 50 de la daira de Maadid) ; 50 artisans et artistes, enquêtes d'opinion auprès de 20 commerçants ainsi que les données fournies par l'office de tourisme. Pour analyser les impacts des festivités sur le site archéologique de la Qal'a, des entrevues semi-dirigées ont été réalisées auprès des divers groupes de sauvegarde du patrimoine et au prés des conférenciers. Une fois la collecte des données terminée, deux principales méthodes analytiques ont été utilisées, la méthode historique avec ses deux versants de critique externe et interne ainsi que l'analyse de contenu et de discours pour le traitement des entrevues de recherche. 


\section{Presentation de la zone d'etude: situation geigraphique et historique}

La Qal'a des Beni Hammad est située dans le village de Béchara, commune de Maadid au Nord-Ouest de la capitale de la région du Hodna : la willaya de M'sila. Géographiquement, la Qal'a est située à $36 \mathrm{Km}$ de cette capitale régionale, à une altitude de 1000 mètres au versant sud du Djbel Maadid à la limite nord des plaines du Hodna. Elle est entourée de monts : au nord par Tekerboust ou Gribissa qui culmine à1458 m, à l'ouest par Gorayan avec ses $1190 \mathrm{~m}$ d'altitude et à l'est par la vallée de l'oued Frej dont les gorges constituent un rempart naturel. Ce qui fait de cette citadelle une vraie forteresse d'une grande difficulté d'accès. D'ailleurs la seule voie de pénétration est au sud, qui constitue un col sinueux longeant la vallée de l'oued et s'ouvrant sur la plaine du Hodna (Voir Fig. 1)

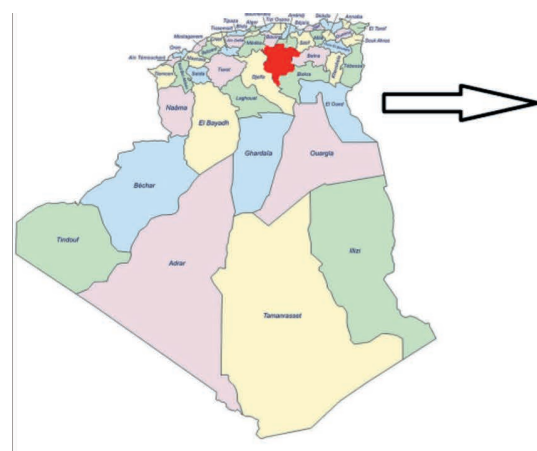

M'sila dans le territoire national algérien

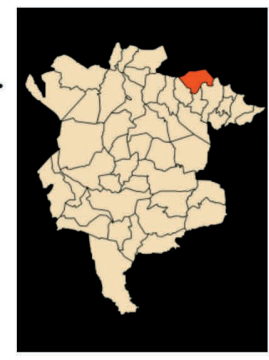

Situation de Maadid dans la wilaya de M'sila

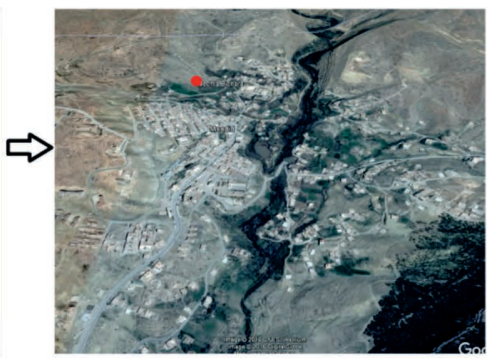

Situation de Béchara dans la commune de Maadid

Fig. 1 - Situation de la Qual'a des Beni Hammad. Source: google earth, 2016

Historiquement, et selon le chroniqueur Ibn Hammad, le lieu d'édification de la Qal'a constituait déjà un ancien établissement romain qui s'appelait fort du Miroir ou château de Kiana (Blanchet 1899 ; De Beylié 1909 ; Kitab el Istikçar). Hamad Ibn Bologhine de la grande tribu berbère des Sanhadja fonda la Qal'a en 1007 et la peupla des habitants de Hamza (l'actuelle ville de Bouira), de M'sila et des Djerawa peuplades du Maghreb. Neuf ans après elle devint, en 1017, la capitale des Hammadide et de tout le Maghreb central, et ce, tout le long du onzième siècle de notre ère. De par sa prospérité économique, culturelle et architecturale, la Qal'a rivalisa avec les grandes capitales maghrébines et devint un centre de commerce qui attira les caravanes de l'orient arabe (Al Muqaddasi, 1950). A ce propos Ibn Khaldoun 
(1852 :43) rapporta que «La Qal'a atteignit bientôt une haute prospérité; sa population s'accrut rapidement et les artisans ainsi que les étudiants s'y rendaient en foule des pays les plus éloignés et des extrémités de l'empire. Cette affluence de voyageurs eut pour cause les grandes ressources que la nouvelle capitale offrait à ceux qui cultivaient les sciences, le commerce et les arts »(Cité par Bourouiba, 1971). Après la ruine de Kairouan, restitua El-Bekri (1859 : 119-120) la Qal'a devint une métropole et un grand centre de commerce qui « attira les caravanes de l'Irac, de l'Egypte, de la Syrie et de toutes les parties du Hidjaz » Le déclin de la ville commença après son abandon par El Mansour en 1090, qui transporta son siège à Béjaia, suite à l'invasion Hilalienne et leurs continuelles déprédations. Depuis, ses palais cessèrent d'être entretenus, notamment suite à l'embarquement de tous les objets de valeur par Yahya, le dernier souverain Hammadide, en 1148, au profit de la nouvelle capitale Bejaia. La Qal'a fut détruite, en partie, par les Almohades en 1152 (El Idrissi, 1866 :100). En 1185, elle fut ruinée par l'almoravide Ali Ibn Ghaniya Depuis, son emplacement est resté vierge de toute autre construction.

\section{Formation architecturale: de la restitution a l'etat actuel}

Couvrant une superficie de 150 hectares, la Qal'a comporte à l'intérieur d'une enceinte fortifiée de $7 \mathrm{Km}$ de périmètre incomplètement démantelée, une quantité importante de monuments architecturaux découverts successivement depuis la fin du XIXe siècle, suite aux premières fouilles incitées par Féraud ${ }^{3}$ (1870), entreprises par Paul Blanchet (1897), jusqu'aux années 1980 date de son classement, par l'UNESCO, comme patrimoine mondial.

Ces recherches menées sur site ont permis de dégager une hypothèse de reconstitution des zones les plus basses du terrain qui se constituaient en quatre parties, dominée chacune par un palais : quartier de la mosquée, celui du palais du Manar, celui du palais des Emirs et du lac et ceux de l'étoile et du salut. En effet, en 1897, seul le minaret de la mosquée était visible (Voir fig.02). Les travaux de fouilles n'avaient concerné qu'une partie du donjon du Manar. En 1908, les fouilles engagées par le général Léon de Beylié avaient

3 D'après le général Beylié (1909) c'est Féraud, dans son histoire des villes de la province de Constantine, qui signala, en premier, à l'attention du monde savant l'intérêt que présentaient, au point de vue historique, les ruines de la Qal'a des Beni Hammad 
permis de mettre en exergue le palais du lac ainsi que le donjon et la partie sud du palais des Emirs appelé aussi du Manar, notamment le bassin avec les quatre demi-colonnes connus sous le nom de «fondations du sultan ».
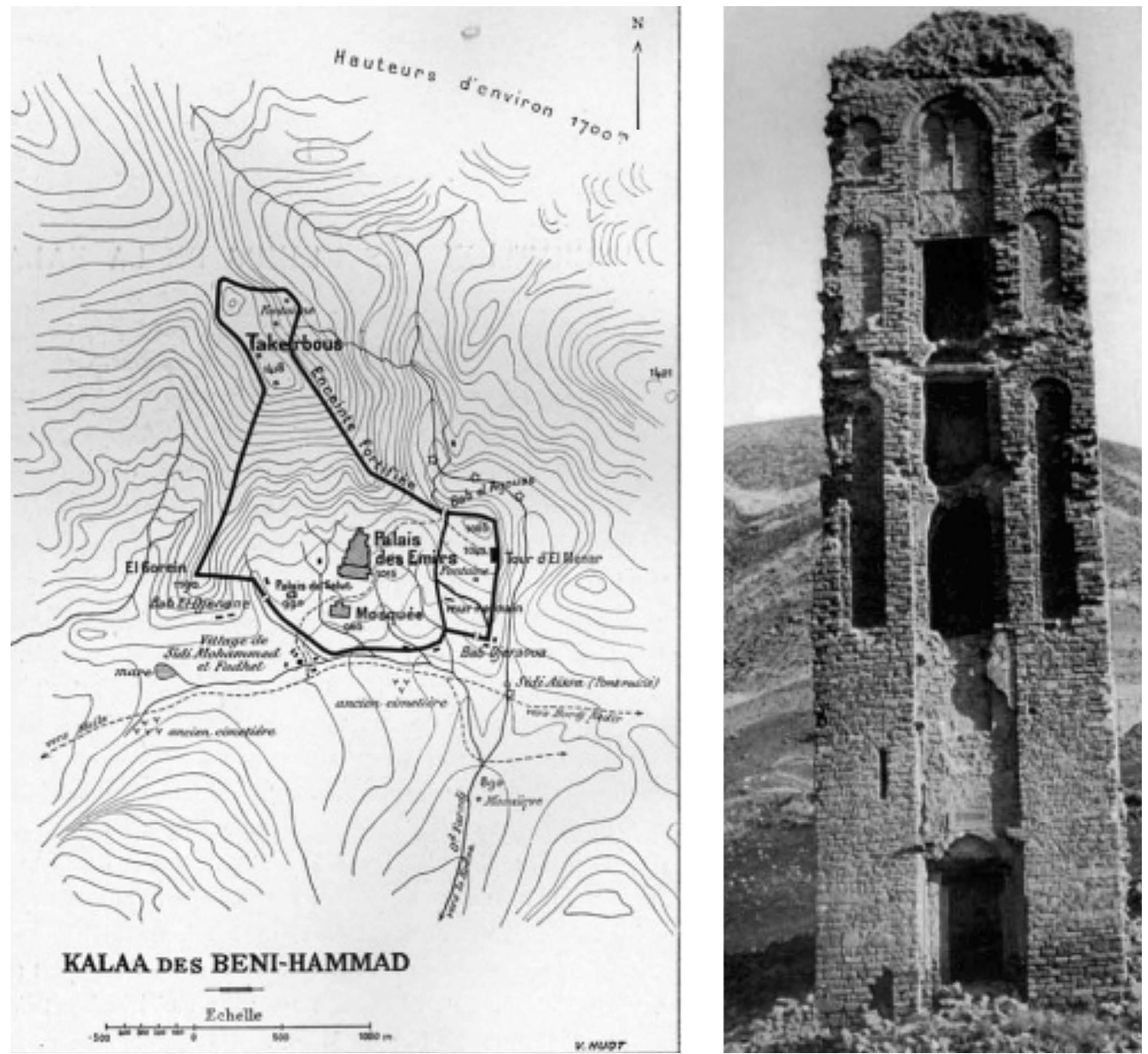

Fig. 2 - Plan de la Qal'a et photo du minaret de la mosquée au début des fouilles. Source : Beylié, 190

En 1951, Golvin avait exhumé en trois étapes (1951, 1952-1956, 1960-1962) le bâtiment central du Manar, la citerne du Nord-ouest de ce palais, son bassin carré avec ses niches intérieures ainsi que le palais du salut (Golvin 1965). Enfin, à partir de 1962, Rachid Bourouiba a mis au jour, en plusieurs étapes, la salle de prière de la mosquée, la partie Est du palais du lac avec la porte en avant corps, ainsi que les traces de la porte Bab-el-Aqwas (Bourouiba 1964 ; 1975). 

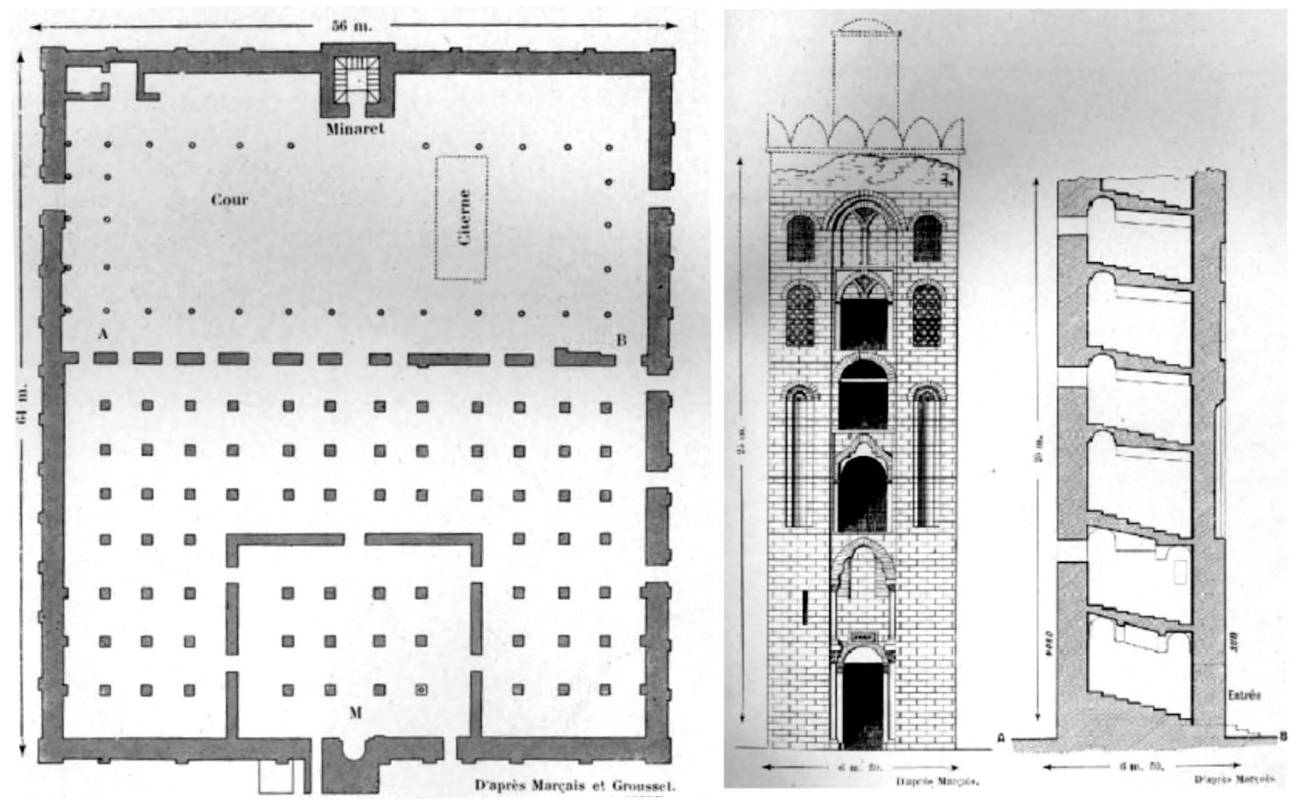

Fig. 3 - Plan de la grande mosquée. Façade et coupe du minaret. Source: Beylié, 1909. Dessins de Georges Marçais et soldat Grousset

\section{1. La grande mosquée et son minaret}

La mosquée de la Qal'a, de 66m de long sur 54m de large, est composée d'une salle de prière rectangulaire mesurant 53,20 sur 34,20 mètres, formée de treize nefs, orientées nord-sud, comportant de huit(08) travées. Cette salle donne sur une cour à portiques avec citerne destinée aux ablutions. Au milieu du coté nord de la cour se dresse un minaret en pierre à base carrée de $6.50 \mathrm{~m}$ de coté et de $25 \mathrm{~m}$ de hauteur. Ce minaret faisait face au Mihrab qui se situait à l'intérieur d'une Maksoura, salle de prière du prince (Voir Fig. 3). Actuellement, de la salle de prière, seuls des murs extérieurs, périphériques de $1.5 \mathrm{~m}$ de large et des poteaux de forme circulaire, reposant sur des dés en maçonnerie subsistent. De la cour, seuls le minaret et la citerne apparaissent encore (voir Fig. 4)

\section{2. Palais du Ménar ou du Fanal}

Situé sur une inclinaison rocheuse abrupte, le palais du Manar appelé aussi fanal a disparu. Il n'en reste aujourd'hui qu'un édifice carré de 22 
$\mathrm{m}$ de coté qui, comme laisse entendre son nom, est un donjon et une tour à signaux. Les fouilles de Beylié avaient montré une tour dont les faces intérieures rappellent celles des palais de Khorsabad de Waraka (Saladin, 1909) comprenant en son sous sol une pièce n'ayant comme issue qu'une ouverture donnant sur la vallée. Vraisemblablement une prison, un magasin ou une salle de rassemblement. Le rez de chaussée, dont le plan ressemble nettement à la salle centrale du palais de la Ziza à Palerme, forme une salle cruciforme entourée de quatre iwans dont un, servait d'entrée. Il était lié à la terrasse supérieure de l'édifice par une rampe qui renforce l'idée des influences persanes sur l'art musulman d'Occident (voir Fig. 5).
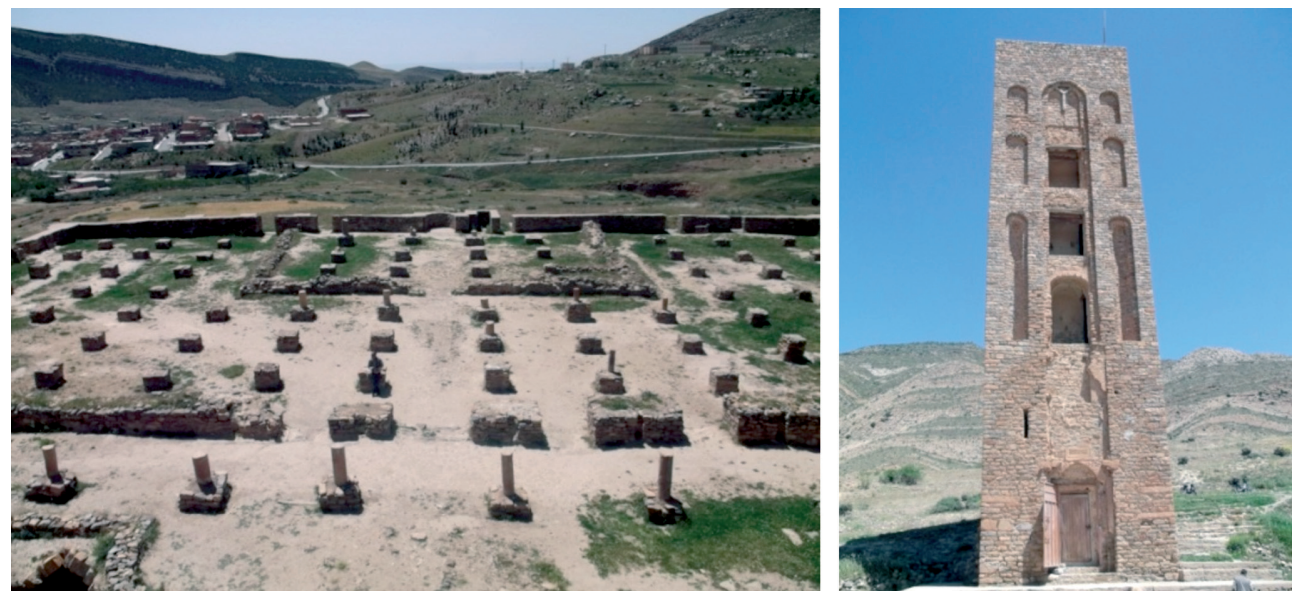

Fig. 4 - Etat actuel de la grande mosquée; façade sud du minaret. Source: Cliché de l'auteur, 2014
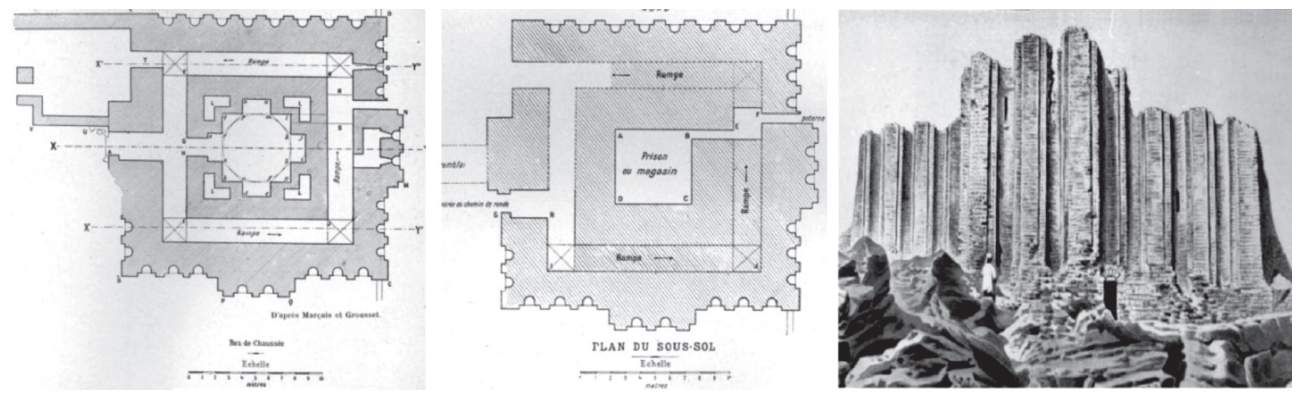

Fig. 5 - Plans du rez de chaussée et du sous sol du Ménar ainsi qu'une vue Est au dessus des gorges du Fredj. Dessins de Georges Marçais et soldat Grousset. Source : De Beylié, 1909

Actuellement, ne subsistent que les contreforts extérieurs sous forme de rainures terminés par des motifs appelés ruches d'abeilles (voir Fig. 6). 

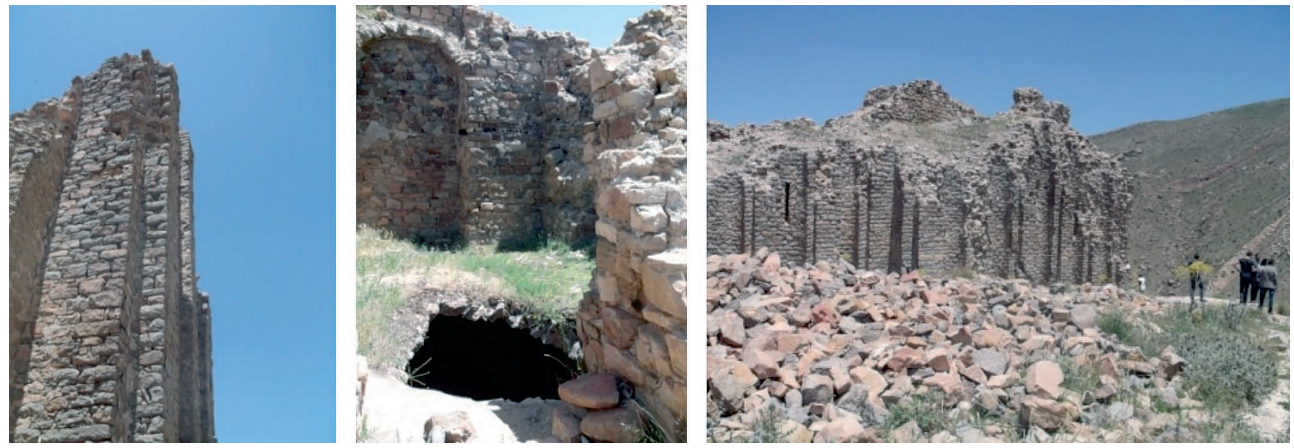

Fig. 6 - Etat actuel du dojon du Ménar; vue Est au dessus des gorges du Fredj et salle du RDC. Source : Cliché de l'auteur, 2014

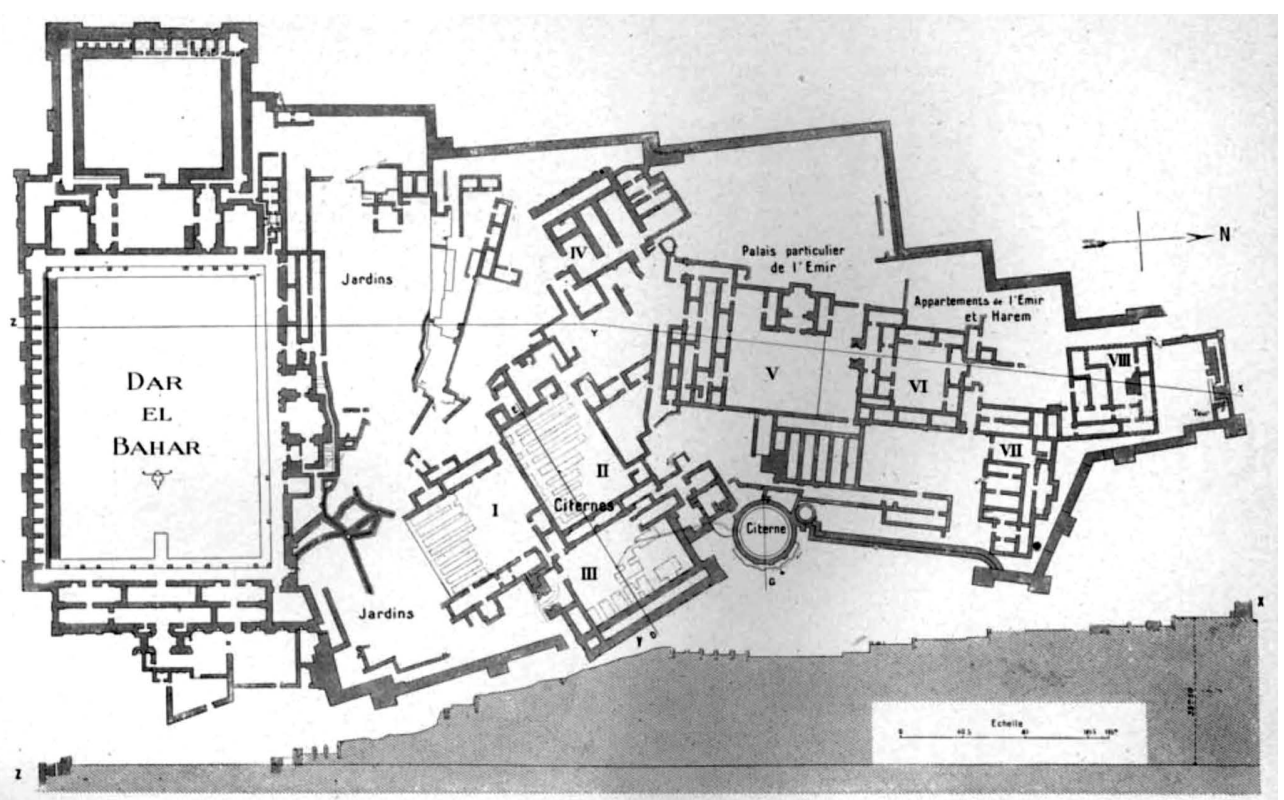

Fig. 7 - Plan du complexe palatial des Emirs. Source : De Beylié, 1909

\section{3. Le palais des Emir et palais du Lac «Dar El Bahar»}

Le palais des Emirs est un complexe palatial composé de trois palais 
distincts, séparés par des pavions, jardins, citernes et habitations (voir Fig. 07). Le premier représentait le palais du gouvernement, lieu des réceptions officielles. Le second était voué à l'habitation privée, le harem. Cependant le plus important d'entre eux, était le palais du lac, nommé Dar el Bahar (Saladin, 1909), vu les deux grands bassins ${ }^{4}$ ou miroirs d'eau qui occupaient le centre de ses deux masses bâties.

Dar El Bahr avait $150 \mathrm{~m}$ de long sur $67 \mathrm{~m}$ de large. La pénétration à ce palais se faisait par l'entremise d'un vestibule coudé qui donnait sur une galerie à portique qui faisait le tour sur les trois cotés de la grande cour. Le côté Nord de la cour donnait sur trois salles. Celui du sud donnait sur une simple chaussée en maçonnerie bordée d'un mur plein. Ce grand bassin était voué aux duels nautiques. La deuxième masse bâtie était vraisemblablement des appartements privés.

Aujourd'hui ne subsiste que les niches demi-circulaires du vestibule ainsi que les murs arrière des trois salles nord ouvrant sur le grand bassin (Voir Fig. 8).
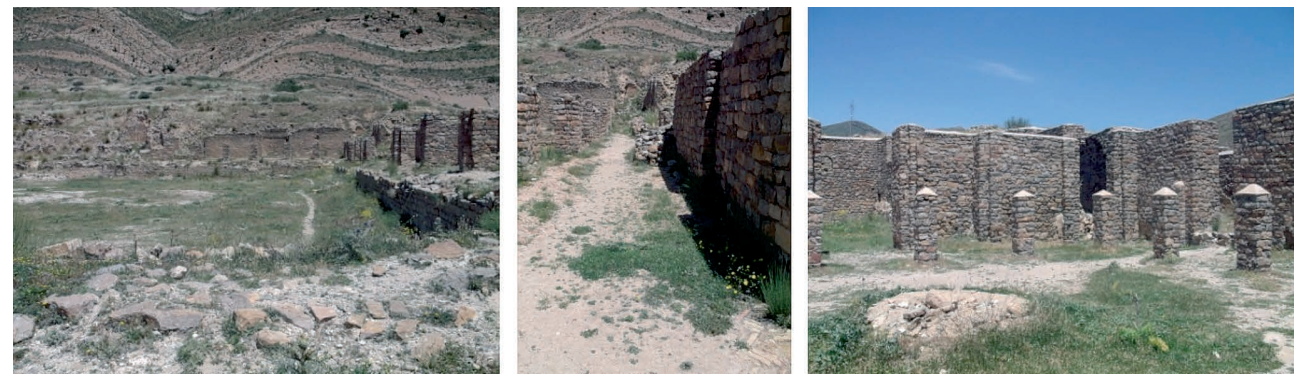

Fig. 8 - De gauche à droite : Etat actuel de la grande cour ou grand bassin; vestibule d'entrée à Dar El Bahar ; la cour centrale du palais du souverain. Source : Cliché de l'auteur, 2014.

\section{4. Palais du Salut}

Situé à proximité de Bab Djenan, le palais du salut est bâti sur une croupe en contrefort. Il est bordé en partie par une muraille. Ce palais semble se présenter comme une kasbah allant en s'évasant au fur et à mesure que l'on descend. Des ruines de la partie supérieures ont été exhumées. De même une maison contenant des silos en forme de vases a été découverte du palais. Ce

4 Kitab El Istikçar donne le récit suivant «Les Benou-Hammad élevèrent à la Kalaa d'importantes constructions, des châteaux bien fortifiés, d'architecture soignée et placés sur les hauteurs. Du nombre était celui du Dar-el-Bahar, au centre du quel était un vaste bassin où avaient lieu des joutes nautiques et où la quantité de liquide nécessaire était amenée de fort loin» 
dernier est formé de quatre pièces et divers réduits. La cour est munie d'un dallage en pierres plates de $15 \mathrm{~m}$ de coté.

\section{Festivites et patrimoinr: nouvelle essence de developement}

Le Petit Robert définit le festival comme étant «une série de représentations ou l'on produit des œuvres d'un art ou d'un artiste». Quant à l'encyclopédie libre, elle semble plus précise en le définissant comme une manifestation à caractère festif, organisée à époque fixe et récurrente, autour d'une activité liée au spectacle, aux arts et loisirs et dont la durée peut varier de plusieurs jours. Cependant la diversité et la complexité du phénomène des festivals sont tellement étendues qu'il est difficile de dresser une définition unique de l'événement (Fléchet \& al., 2013). En effet, Si `à l' origine les festivals étaient uniquement consacrés à la musique classique (Benito, 2003 :5 ; Diamantaki, $2010: 38$ ), ils sont aujourd'hui d'une grande variété allant du cinéma à la bande dessinée, aux musiques actuelles, au théâtre à la danse et arts de la rue. Dans tout ce pèle mêle conceptuel, Pascal Ory (2013 : 19-32) tente de le définir, en indiquant que ce terme d'origine anglophone, ne prend son sens actuel qu'à partir du moment « où il est adopté hors de son pays d'origine, pour un usage qui dépasse le sens original et dont peuvent rendre compte d'autres termes d'acceptation locale » En dépit de cette grande diversité, les festivals peuvent être classés selon Mercier et Bouchard (2014 : 17) en quatre grandes catégories non exhaustives: De création, de diffusion, d'image, et touristiques.

Fondés autour d'un projet artistique, les festivals de création ont pour but principal la promotion culturelle, via la production de spectacles originaux. Lorsque les festivals offrent à des publics la possibilité de voir des spectacles dont ils ne peuvent bénéficier le reste de l'année, faute de logistique adéquate, ils sont appelés de diffusion. Quant aux festivals d'images, comme leur nom l'indique, ils sont organisés par des collectivités locales soucieuses de promouvoir et de valoriser l'identité et l'image de leurs sites. La dernière catégorie ou festival touristique vise à mettre en exergue un élément du patrimoine par l'organisation d'un événement à la dois festif et culturel.

Au niveau mondial, spécifiquement en France, les festivals avaient commencé à prendre de l'ampleur dès la fin de la première moitié du XIXè siècle, notamment avec le mouvement orphéonique, cette fête musicale populaire à vocation charitable et politique. Ils ont atteint une grande portée médiatique durant la guerre froide, utilisés alors dans le grand enjeu diplomatique et dans le cadre concurrentiel entre les deux blocs. Dans les pays maghrébins, 
les premiers festivals datent du début du XXe siècle. A titre indicatif, le premier festival tunisien, celui de Carthage date de 1906. Cependant, cette notion est restée dans ces pays, pendant longtemps au stade théorique, Ddu fait qu'elle était considérée comme un concept élitiste et hors de propos en face de la pauvreté, la privatisation et les inégalités dans la vie quotidienne et par conséquent faisait mauvais ménage avec les conditions sociales et finit par engendrer un conflit d'éthique (Labaal \& Zérouala, 1997).

En Algérie, conscients de leur mission et contribution au rayonnement culturel et artistique du pays, l'office national de la culture et de l'information (ONCI) en collaboration avec le Commissariat national du festival (CNF) organisèrent dés la première décennie après l'indépendance plusieurs festivals conçus comme de véritables rendez-vous culturels et artistiques de première catégorie, parmi lesquels nous citons, dans une liste non exhaustive, Fête du Corail d'El Kala, Fête de la datte ou Moussem de Taghit, festival de Sebeiba à Djanet, la fête du bijoux Kabyl à Ath Yeni (1995), festival de la poterie à Maatkas (2010), le festival du tapis à Ait Hicham, la fête de la robe Kabyle à Tizi Ouzou (2010), le festival Arabo Africain de la danse folklorique à Tizi Ouzou (2005). Et dans une perspective de valorisation $\mathrm{du}$ patrimoine culturel et touristique des vestiges historiques notamment les théâtres et les thermes romains ainsi que les palais berbères, plusieurs festivals ont pu voir le jour tel que le festival international de Timgad crée en 1967 et plus récemment la fête du printemps de la Qal'a des Bénni Hammad. Le patrimoine archéologique est alors considéré comme l'incarnation visible et palpable de la mémoire des territoires, et par là même de leur identité. En effet, depuis la fin des années 1990 et à l'instar d'un bon nombre des pays du monde (Négrier et Jourda, 2007 ; Autissier, 2008), un regain d'intérêt pour l'espace local, en Algérie, s'est accompagné d'un nouveau regard sur la notion du patrimoine notamment archéologique, celui-ci est de plus en plus perçu comme un créateur de richesse et source d'attractivité pour les territoires (Idir, 2013 :2). La politique patrimoniale algérienne s'est vue renforcée par la restructuration de ses outils en déconcentrant les pouvoirs et responsabilités en faveur des collectivités et associations locales. Ainsi le passage d'une gestion centralisée du patrimoine à une logique de déconcentration s'est matérialisé par la mise en place d'agences de gestion des biens culturels à travers toutes les régions du pays (Assam-Baloul, 2013). Le but est la valorisation du patrimoine en tant que composante essentielle non seulement dans le développement économique, mais aussi culturel et social. La particularité du patrimoine local concourt à produire une image de marque, dont les acteurs de l'aménagement et du tourisme se saisissent 
comme favorable à assurer et à consolider l'attractivité du territoire. L'enjeu s'avère très important et d'urgence en Algérie, ce pays où l'économie du tourisme à la différence de ses voisins ${ }^{5}$ ne représente encore qu'un faible pourcentage de l'ordre de 2,3\% du PIB (hors hydrocarbures) et n'occupe que $1,8 \%$ de la population totale active. Le dynamisme du territoire du Hodna, notamment celui de la région de Maadid repose donc sur la capacité de la Qal'a des bénni Hammad à rester attractive tant pour les acteurs économiques que pour les populations résidentes.

\section{Fete du printrmps un levier economique de la dairda de Maadid?}

\section{1. Contexte socio-economique et politique de la naissance de la fête du printemps}

En 1980, la Qal'a des benni Hammad intégra la liste du patrimoine mondial de l'UNESCO. Par cette heureuse intégration, la commune de Maadid espérait alors un destin fait de privilèges et de nouveaux pouvoirs. Or les directives de la nouvelle stratégie de développement touristique de l'époque ${ }^{6}$, définie conformément aux orientations de la charte nationale de 1976, stipulaient comme objectif primordial la satisfaction des besoins sociaux de la population nationale. Les loisirs et la détente des travailleurs, pendant leurs congés payés, notamment en bord de mer, constituaient des objectifs phares de la nouvelle politique sociale. Le climatique, le camping et le tourisme culturel (découverte des sites archéologiques) restaient bien en marge. Malgré cette stratégie, le site de la Qal'a accueillait un petit festival de chant local, et ce, jusqu'en 1987. Durant les années 1990, sous le double effet économicopolitique dû, d'une part à la conjoncture économique défavorable engendrée par la baisse des ressources financières liées à la dégradation des prix du pétrole de 1986, d'autre part à la mauvaise situation sécuritaire de l'Algérie dû au terrorisme des années 1990-2000, la promotion touristique avait connu

\footnotetext{
5 En 2004, selon Idir (2013), les contributions du secteur touristique à l'économie marocaine, tunisienne et égyptienne sont respectivement $5,1 \%, 7,6 \%$ et $7,1 \%$

6 Dans le but de hisser l'Algérie au rang des destinations internationales, la charte du tourisme de 1966 avait accordé la priorité au tourisme international selon les deux facettes balnéaire et saharienne. Cependant avec l'amélioration du niveau de vie des algériens durant la décade des années 1970 ainsi que la forte occupation des lits d'hôtels classés par les nationaux, qui est passée de 45000 en 1969 à 486000 en 1978, avaient incité le ministère du tourisme à changer de cap et donner la priorité à la demande nationale.
} 
une dégringolade léthargique sur tous les plans (Boukhelifa, 2007 ; Boudjani, 2008). Maadid était l'une des localités du Hodna qui avait le plus souffert du terrorisme de la décennie noire. La Qal'a présentait, de par sa situation isolée et sa latitude à plus de $1000 \mathrm{~m}$, une destination dangereuse. Quant aux festivités, elles étaient considérées comme acte prohibé par les groupes terroristes et par conséquent sont évitées, sous toutes leurs formes, par les autochtones. Cette conjoncture politique avait de lourdes conséquences sur le plan économique et socio culturel de la daïra de Maadid, qui est devenue

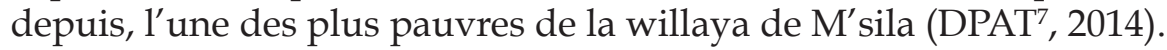

A la fin des années 2000, après l'apaisement de la situation sécuritaire d'une part, et après l'adoption du plan «Horizon 2005» qui visait à assouplir la réglementation pour les investissements étrangers et locaux dans le but d'attirer un grand nombre de touristes étrangers et locaux (Scott \& Jafari, 2010: 281) d'autre part, Maadid, connue par ses larges vergers et beaux paysages naturels, commença à reconquérir de nouveau, pendant la saison printanière, les familles des localités de Béchara, Rachana, Djaouna, Zitoun et $\mathrm{d}^{\prime} \mathrm{El}$ Ghil. Rite qui s'est rapidement étendue à une grande majorité des familles de tout le Hodna oriental qui venaient chercher divertissement et épanouissement. Dans le but de faire renaitre cette localité, en mettant à profit l'apport économique de cette masse considérable de visiteurs, qui prenait de l'ampleur d'une année à l'autre, les autorités locales, notamment les directions de la culture et du tourisme en collaboration avec la direction de la jeunesse et du sport ainsi que la société civile, via le mouvement associatif, lancèrent en 2014 les festivités du printemps de la Qal'a ${ }^{8}$. Cette invention culturelle s'intègre dans un nouveau processus de renaissance territoriale sous la forme de mise en scène de la mémoire et du patrimoine.

\section{2. Specivites culturelles de la fete du printemps}

Dans leurs pluralités, les festivals ont trouvé une place privilégiée au sein des politiques publiques de relance du patrimoine via la culture. Cette dernière a été pendant très longtemps perçue comme un domaine ne pouvant pas être soumis aux critères économiques, considérée même comme source d'activité limitée voire improductive (Smahi \& Boumrar, 2013). En effet, depuis la fin

\footnotetext{
7 Direction de la planification et de l'aménagement du territoire, willaya de M'sila

8 En 2014, cette fête était placée sous le thème: Histoire de notre nation source de notre unité. En 2015, sous le thème: patrimoine et régions et en 2017, sous le thème: Le Patrimoine un levier économique solide pour la région.
} 
des années 2000, le regard des décideurs et de la société civile avaient changé. Le couple patrimoine/ tourisme et culture de festivités est reconnu de plus en plus comme une ressource humaine et matérielle capable de mobiliser et de dynamiser les populations locales. L'organisation d'événements culturels et touristiques était considérée comme catalyseur de développement de l'image, de la notoriété et des retombées économiques d'une destination. C'est dans cette visée qu'a été institué la fête du printemps de la Qal'a. Cette dernière est une festivité folklorique pluridisciplinaire qui dure trois jours. Elle commence le 18 avril, coïncidant ainsi avec la commémoration de la journée mondiale du patrimoine. Le but de cette pluridisciplinarité est, de proposer, d'une part, une animation locale continue et de limiter, d'autre part, au maximum les frais d'installation. La fête du printemps est devenue un symbole voué à promouvoir l'identité culturelle locale des Modhadi ${ }^{9}$. La fête de la Qal'a est organisée autour de quatre grands axes :

\section{Le premier axe est dédié aux pratiques artistiques}

Il s'agit de chants folkloriques de la région, de lectures poétiques, de pièces théâtrales, de shows humoristiques, feu de gags, d'humour et d'énergie dans une animation de sketches où défilent des personnages aussi drôles que touchantes, ainsi qu'à la programmes de distractions pour enfants ${ }^{10}$. A titre d'exemple nous citons les fameux concerts de chant des groupes «Billet de voyage» de Smail Yles, «Wissam» de l'association de la promotion de la culture et des arts du Hodna, «poésie Hodnéennes» pour la promotion des anciens chants poétiques Hodni de Merniz ainsi que le célèbre groupe de Lyamine Khamissa dans la catégorie des comiques.

\section{Le deuxième axe est consacré aux concours socioculturels}

Les plus importants d'entre eux sont ceux de la gastronomie et de la photographie. Le concours gastronomique est dédié à tous les cuisiniers professionnels qualifiés. Les concepts culinaires mis en relief ont été rattachés à la créativité et la modernité ainsi qu'aux plats traditionnels algériens. Quant au concours de la photographie, il était ouvert à tout photographe, sans condition d'âge, de sexe ou de statut

${ }^{9}$ Habitants de toute la région des localités de Béchara, Rachana, Djaouna, Zitoun et d'El Ghil 10 Neuf (09) groupes de chants folkloriques, plusieurs artistes appartenant à six (06) groupes de théâtre, et 10 groupes d'animations réservées spécialement aux enfants. 
Le troisième axe est dédié aux expositions artisanales locales et nationales Une douzaine de wilayas ${ }^{11}$ (départements) contribuent chaque année à cette exposition qui se veut un aperçu sur la richesse, la beauté et la diversité de la tapisserie, bijouterie et poterie algérienne. Les collections présentées comprennent des tissages de plusieurs régions du pays, notamment le tapis de Djeballa (région de Jijel), de Mergoum (régions Maadid, Zitoun, Melouza) ; de Hanbal (région de Boutaleb); des châles appelés «khomri» de la région d'El-Oued et de Ghardaïa ainsi que des costumes kabyles et Chaoui. À côté trônent des ouvres de poterie et bijouterie artisanales, décorées de motifs très variés dans une palette haute en couleurs.

\section{Le quatrième axe est consacré aux conférences, séminaires et expositions muséales}

Un cycle de conférences «histoire et archéologie du site de la Qal'a des Benni Hammad» organisé conjointement par la direction de la culture et le département d'Histoire des sciences humaines et sociales de l'université de M'sila, met en valeur les apports scientifiques des deux institutions. A chaque séance un universitaire, chercheur ou spécialiste, présentent soit une conférence magistrale sur le thème étudié dans le cadre du séminaire annuel organisé sous la thématique "Qal'a des Benni Hammad: un patrimoine à revaloriser», soit un document phare sorti exceptionnellement des collections du musée de Maadid. Ce cycle, via les spécialistes de l'art de bâtir, de fouille et de conservation, vise à informer les étudiants qui auront un jour en charge le patrimoine sur les apports et limites de la conservation-restauration quant à l'étude du patrimoine archéologique et celle des œuvres d'art. Les séances abordent l'identification des matériaux en présence, les principaux processus d'altérations des objets selon leur typologie, ainsi que des conseils de manipulation. Les présentations sont suivies d'un temps d'échanges où les spécialistes et profanes posent des questions, ce qui crée un climat de convivialité entre les chercheurs et le public instruit.

\section{3. Impacts economiques de la fete du printemps sur la localite de Maadid}

Du fait de sa jeunesse, la fête du printemps de la Qal'a n'attire qu'un infime pourcentage du public de provenance nationale (quelques familles des

11 Wilaya d'Adrar, Ghardaia, Khenchela, Biskra, Tiaret, Tizi Ouzou, Béjaia, Batna, Djelfa, Sidi Belabbes, Guelma, El Oued et [ije] 
wilaya de Biskra et de Batna ont été recensées). Son public est exclusivement local à la région du Hodna oriental. De provenance de M'sila, Selmane, Ouled addi Legbala, Berhoum, Belaiba et quelques fois de Mcif, Barika et Mdoukel.(Voir Fig. 9)

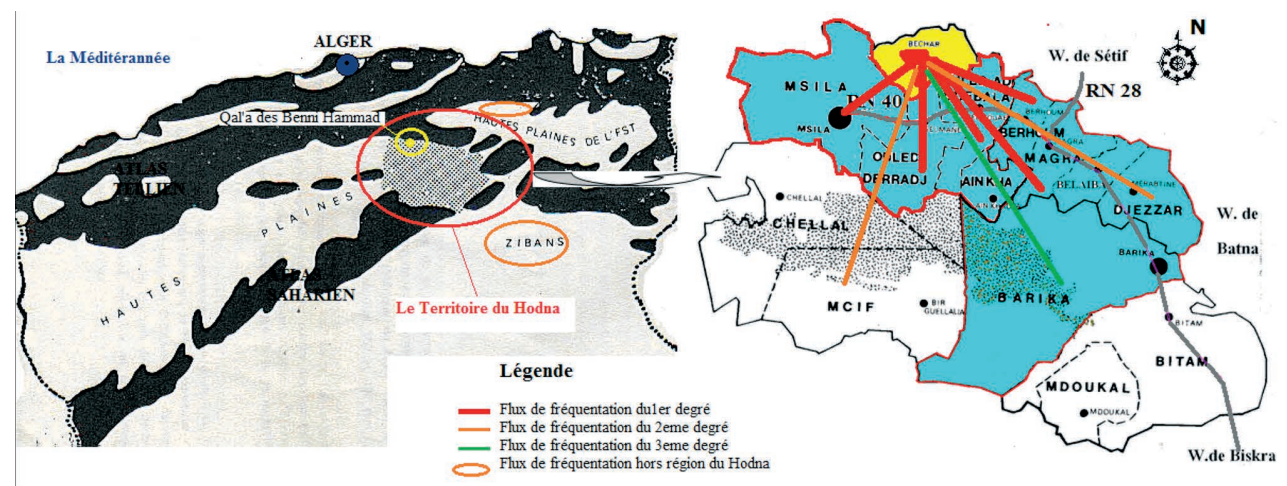

Fig. 9 - Provenance et Flux de fréquentation de la Qal'a des benni Hammad. Source: Auteurs, 2016

Etant donné les difficultés à comptabiliser, même approximativement le nombre de personnes fréquentant la fête du printemps, les responsables locaux estiment que les chiffres varient entre 500 à 700 personnes par jour, dont prés de 470 visiteurs grand public, 50 artisans, 80 excursionnistes et 100 conférenciers. De manière schématique et selon les mêmes sources, les artisans-commerçants comptent pour environ $7.14 \%$, les conférenciers représentent $14.28 \%$ alors que le grand public compte $67.14 \%$ du total des visiteurs dont $11.42 \%$ d'excursion d'écoliers.

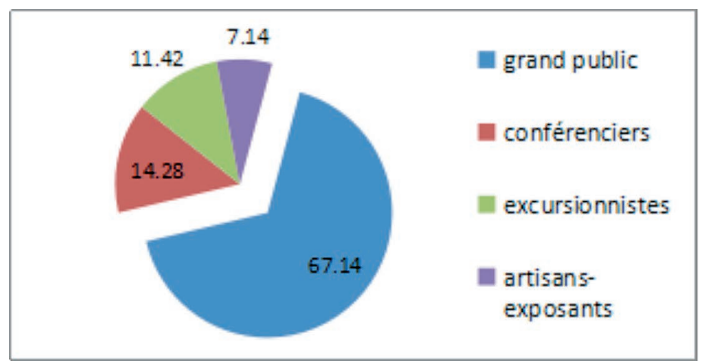

Fig. 10 - Répartition des visiteurs de la fête du printemps de la Qal'a des benni Hammad. Source: Auteurs, 2016
Les tranches d'âges moyennes sont très représentées dans cette fête. Dans l'ensemble, le public apparaît (selon de simples estimations) plus féminin, avec une nette dominance des moins de dix huit ans. Si en Europe, la surreprésentation des diplômés aux festivals est un trait majeur à l'instar de tout autre public de la culture (Négrier, 2009 :70), l'enquête a montré que le niveau 
des festivaliers de la Qal'a est très variable. S'agissant d'une fréquentation familiale sous égide patriarcale, il a été jugé plus judicieux de procéder à l'enquête par famille. Les résultats ont révélé que plus de la moitié des pères de familles fréquentant le festival exercent des activités libérales supérieures ${ }^{12}$ (soit 51\%). Les visiteurs, commerçants de métiers, constituent la deuxième tranche avec un pourcentage de $33 \%$ dont $19 \%$ ayant un diplôme universitaire. Suivis par les employés du secteur public, tout corps confondus, enregistrant ainsi un taux de $15 \%$, le reste, soit $1 \%$ sont des pères de familles déclarant un emploi temporaire. Ils sont généralement originaires des localités de Béchara et de Maadid (voir Fig. 11).

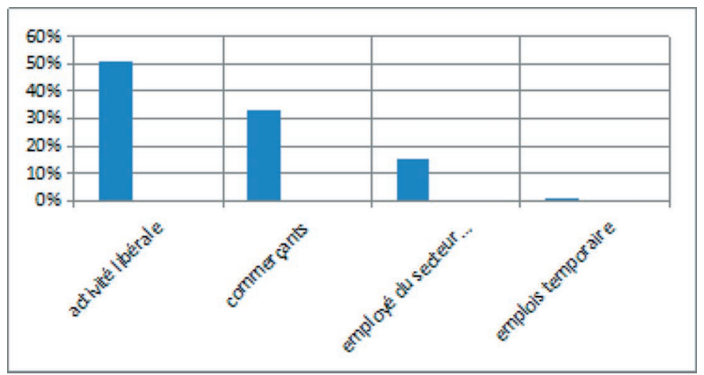

Fig. 11 - Niveaux et activité du grand public fréquentant de la fête du printemps de la Qal'a des Benni Hammad. Source: Auteurs, 2016

La majorité des visiteurs grand public est présente pour une journée, soit un taux de $92 \%$. De la minorité de $8 \%$ qui réside pour le festival, $2 \%$ est en hébergement marchand contre 6 $\%$ environ en hébergement non marchand (Voir Fig. 12 a).

Les dépenses sont variables d'un poste à l'autre. Celles liées à la restauration et dans les cafés arrivent en tête avec un taux de $50 \%$, suivent ensuite les dépenses liées au commerce de miel, souvenirs, poterie, tapisserie (respectivement $20 \%, 15 \%, 8 \%$ et $5 \%$ ). Puis celles liées à l'hébergement Voir Fig. 12b). Par manque de statistiques nous ne sauront donner avec exactitude le chiffe d'affaire engendré par les visiteurs grand public, cependant d'après l'enquête au prés des visiteurs générateurs d'impact, la dépense moyenne par jour est de 1000 à 4500 DA par famille, ce qui fait grimper le chiffre d'affaire calculé, par simple multiplication du nombre total estimé des visiteurs, à 3150000 DA. Les commerçants et artisans de leur part, expliquent que cet afflux massif des visiteurs pendant ces trois journées que dure la fête du printemps, fait grimper leurs chiffre d'affaire au double, sans donner de précision.

Pendant ces jours de festivités que dure le fête du printemps, les localités de Béchara et de Maadid se retrouvent totalement transformées. La fête du

12 Par activité libérale supérieure est compris médecins, avocats, architectes, ingénieurs, notaires, comptables...etc 
printemps constitue un lieu d'échange et de rencontre. Les habitants montrent une grande satisfaction. Des liens s'instaurent entre grand public et habitants de la région. De leurs cotés les artisans-commerçants exposants nouent des liens économiques importants avec les futurs acheteurs qui deviennent des clients permanents. Les lieux sont investis, les placent du parking du centre ville et même dans le périmètre immédiat de la Qal'a affichent complet, ce qui constitue une très grande source économique pour les jeunes chargés du gardiennage de ses parkings le plus souvent illicites. De sa part, le site historique de la citadelle regorge de marchands ambulants (de cigarettes, de friandises, de fleurs, de souvenirs), d'excursionnistes, d'écoliers et de familles entières.

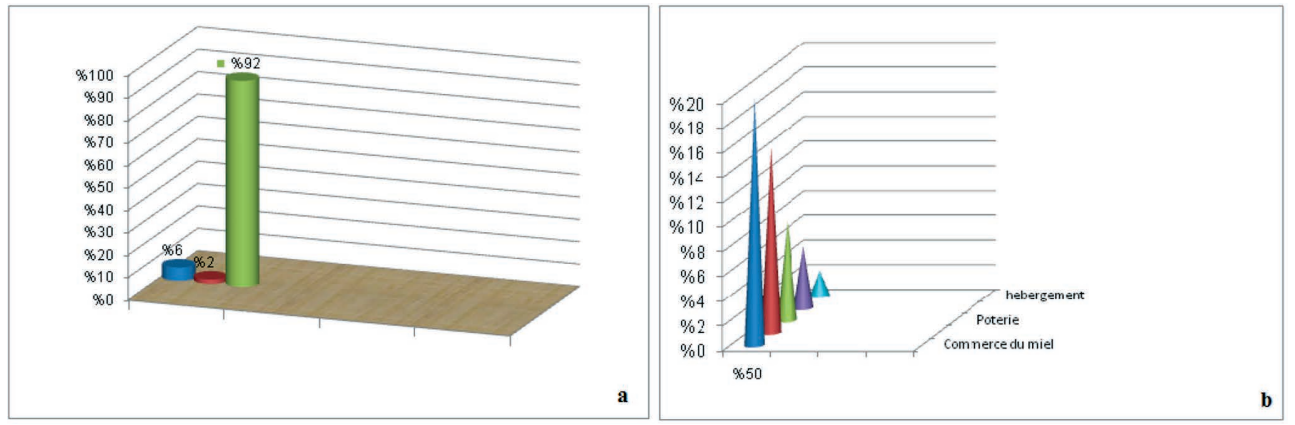

Fig. 12 - Hébergement et taux de consommation du grand public de la fête du printemps. Source: Auteurs, 2016

\section{Fete du printemps, quel impact sur le site archeologique?}

Dans leurs majorités, les autorités locales de la wilaya de M'sila perçoivent le tourisme comme élément essentiel à la vitalité économique de la région Béchara -Maadid et même de tout le Hodna oriental dans la mesure où le tourisme régional et le patrimoine de la Qal'a s'alimentent réciproquement. Cependant, cette relation binaire n'est pas toujours positive, et enregistre beaucoup d'effets négatifs. En effet, la fête du printemps est vue aussi bien par les habitants que par les festivaliers comme un événement de divertissement. Le site de la Qal'a de par sa verdure et son étendue, et en l'absence de tout contrôle des services de sauvegarde patrimoniaux, est vu comme le meilleur endroit où passer les après midi, après avoir vu les expositions, assister aux conférences et faire des achats. La majorité des familles consomment leurs repas, achetés sur place, non pas dans les restaurants, mais sur le site de la citadelle. Deux endroits semblent très appréciés pour le «pique- 
nique sauvage» de ces festivaliers non éduqués au respect du patrimoine : les ruines de la mosquée et de sa cour, ainsi que le palais du lac. Les murs de pourtour et les assises des poteaux de la mosquée sont utilisés par les marchands ambulants comme éléments d'étalage de leurs produits et par les festivaliers comme chaises (voir Fig 13. 1). Le minaret à son tour, n'est pas épargné et, malgré son état déplorable qui interdit toute utilisation massive, voit son escalier utilisé par des groupes formés de plusieurs personnes à la fois, désirant atteindre le sommet pour avoir une vue panoramique sur le site archéologique ainsi que sur toute la localité de Maadid (voir Fig 13. 2). Par inconscience, le seul gardien de tout le site, qui ne peut surveiller que le minaret, croit en se pliant aux exigences des festivaliers, en leurs permettant de monter sur le toit du minaret, accroitre l'attractivité touristique du site. De leurs cotés ces derniers, notamment les jeunes d'entre eux, inscrivent leurs noms partout sur les vielles pierres du plus important vestige de la citadelle (voir Fig 14. 1).
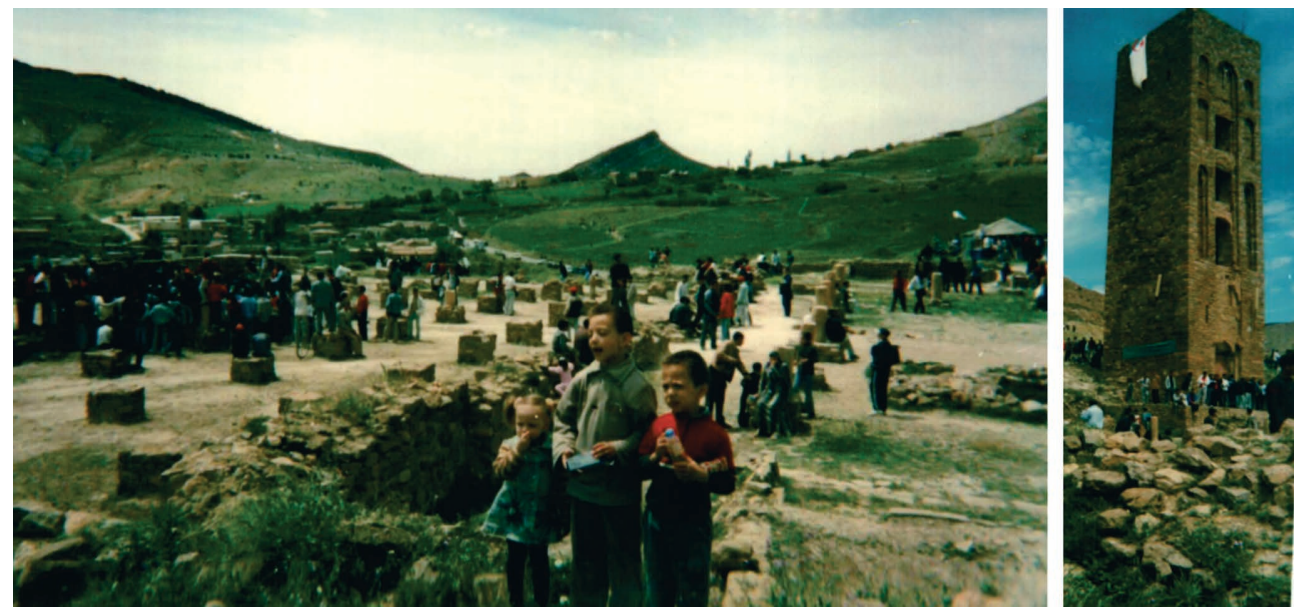

Fig. 13 - De gauche à droite: utilisation des assises de poteaux comme chaises et étalage des produits sur les murs périphériques de la mosquée ; foule de festivalier en attente pour monter en haut du minaret. Source: Auteurs

Dans le but de désenclaver les villages montagneux limitrophes de la Qal'a, une voie mécanique reliant la wilaya de Bordj à $\mathrm{M}^{\prime}$ sila a été réalisée. Certes ce chemin départemental avait permis aux populations de ces patelins, entre autres, de profiter des festivités, cependant cette voie réalisée à deux mètres du minaret avait donné lieu à des poussées souterraines et avait fini par provoquer des fissurations dans les parois du minaret (voir Fig 14.2). Des fissurations qui se sont aggravées suite aux vibrations engendrées par le 
passage des véhicules. De même un parking illicite situé au coté gauche du minaret à quelques mètres de l'ancienne porte des jardins « Bab Djenane » de la citadelle, fait apparition à chaque printemps et plus spécialement à chaque festivité, facilitant ainsi l'arrivée des excursionnistes. Ces derniers, faute d'existence de parcours balisés, piétinent les dallages ancestraux des différents palais, mettant ainsi en péril un précieux patrimoine (Voir fig 14.3).
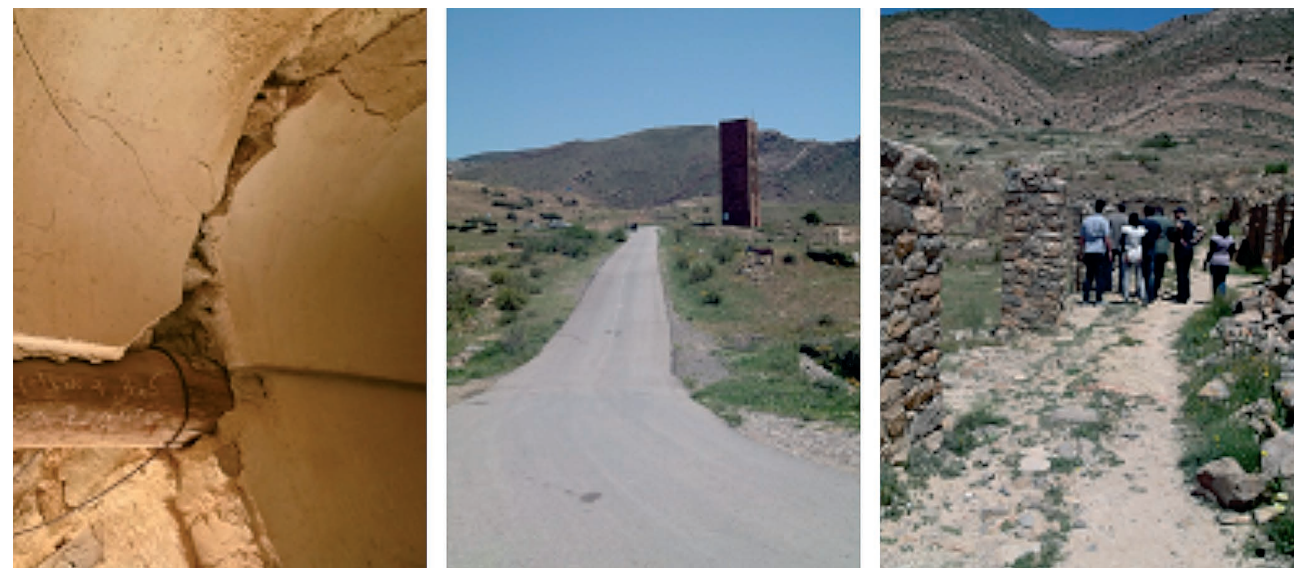

Fig. 14 - De gauche à droite: 1. Fissurations des parois internes du minaret et utilisation de graffitis; 2 . Chemin départemental nº; 3. Piétinement des dallages par les excursionnistes. Source: Auteurs, 2014
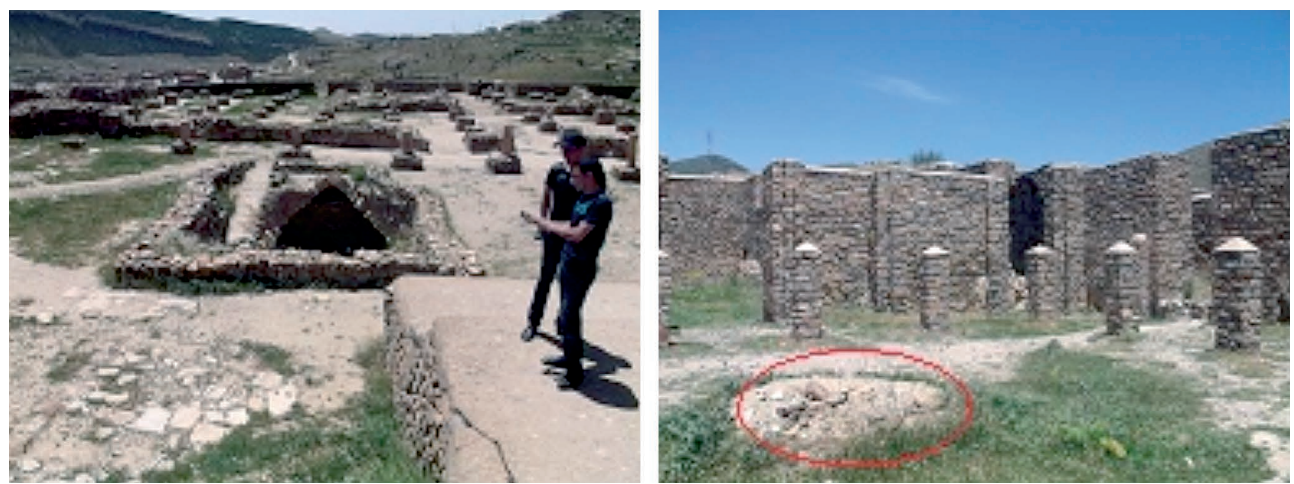

Fig. 15 - De gauche à droite: 1. citerne de la mosquée; 2. Fontaine du sultan après enterrement. Source: Auteurs, 2014

D'un autre coté, l'ouverture de certains espaces vulnérables aux excursionnistes a précipité le processus de dégradation de la citerne à ciel ouvert de la mosquée (Voir fig 15.1) et la probable remise d'armes du donjon 
du Manar. Par insuffisance du potentiel humain et matériel en matière de gardiennage, des matériaux de construction et des éléments architectoniques notamment les marbres rouges de la salle de réception du palais du Manar ont été à plusieurs reprises dépouillés. A titre d'exemple, la fontaine du sultan, bassin de pierre au lion, qui est une vasque de pierre se trouvant au centre du riche complexe ouest du palais du Manâr a été volontairement remise sous sol à défaut de pouvoir la protéger des actes de vandalisme et de vols (Voir Fig 15. 2 et Fig 16).
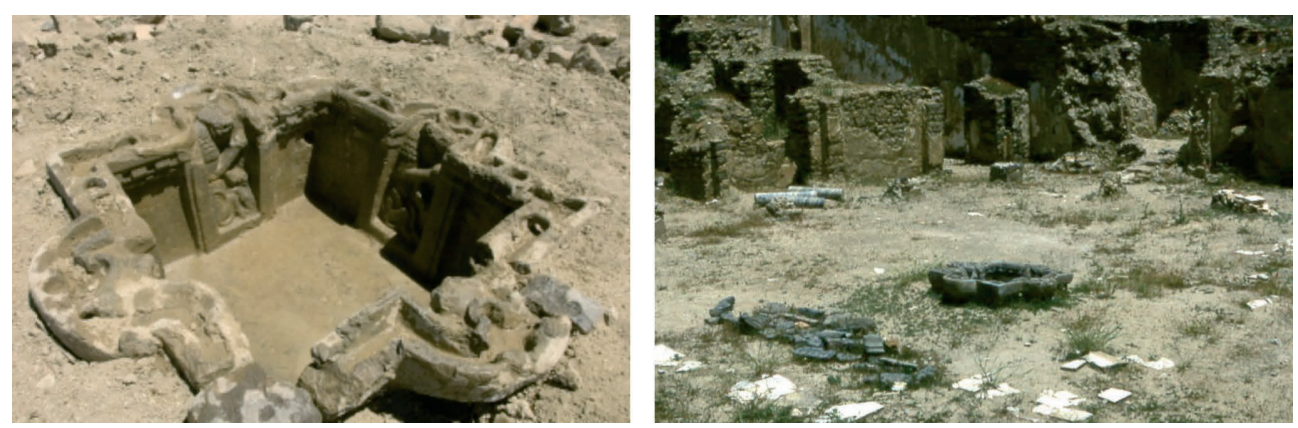

Fig. 16 - Bassin de pierre au lion. Source: www.qantara-med.org

\section{Conclusion}

L'impact de la fête du printemps sur la commune de Maadid et particulièrement de la localité de Béchara est certain, notamment en matière d'image, de développement économique et touristique. Les retombées réelles que les activités qu' elle initie sont bien clairs et évidents. Cette fête a permis, grâce au programme tracé en collaboration avec la société civile, via le mouvement associatif et les pouvoirs public, la revalorisation des métiers traditionnels (poterie, tapisserie et bijouterie). En dépit que cette fête n'a pas encore, faute de maturité, garanti d'emplois permanents, cependant elle a permis aux petits commerçants ambulants et fixes de faire des bénéfices sur les produits vendus, comme elle a permis de faire revivre cette région longtemps enclavée et délaissée. Si de par le monde, les festivals sont considérés comme d'importants facteurs contribuant à la restauration des monuments historiques dans lesquels ils se déroulent, tel que les cas du festival de "Saint-Guilhemle-Désert » qui a été crée en 1972 pour permettre la réparation de son monumental orgue ou du festival «Sinfonia » en Périgord crée en 1990 
pour rénover l'église de Saint-Jean-de-Côle, la fête du printemps, faute d'une réglementation adéquate en symbiose avec nouveau le contexte socio économique que connait l'Algérie, a eu des répercussions négatives sur le site archéologique de la citadelle des Benni Hammad. En effet, le manque de coordination entre les délégations des ministères de la culture de l'aménagement du territoire, de l'Environnement et du tourisme ainsi que l'Agence Nationale d'Archéologie ont conduit à une gestion matérielle irraisonnée de la ressource patrimoniale. La mise en tourisme de la citadelle via la festivité du printemps a donné des effets pervers de dommage des ressources patrimoniales. La cause principale peut être attribuée à deux grands facteurs : l'insuffisance de protection du site et la gratuité de son entrée ainsi qu'au laxisme de l'état en matière d'éducation envers le patrimoine, car le politique du patrimoine comme l'avait bien définit De Mirieu de Labarre, (2004) ne dépend pas seulement d'un code et de son perfectionnement; il est aussi liée à la connaissance que les peuples, les citoyens et les élus ont de l'histoire. En effet « l'absence de conscience et de science historique n'est, peut-on écrire, que la ruine de l'âme et de l'identité culturelle » Ainsi une bonne éducation en faveur du patrimoine, via des sensibilisations continuelles des touristes d'une façon générale et des jeunes excursionnistes, d'une façon particulière, ne peut être que bénéfique à long terme, car les enfants d'aujourd'hui sont les touristes de demain. D'une manière générale, le tourisme est vu par les autorités locales comme source de fierté, c'est un élément primordial pour la revalorisation sociale et économique de toute la région de Maadid, mais reste pour les groupes militants pour la sauvegarde du patrimoine et l'ordre local des architectes comme source d'inquiétude et de menace, car qualifié de trop événementiel et trop concentré dans le périmètre de la citadelle, d'autant plus qu'il se fait en l'absence d'une réglementation et d'une gestion qui ignore l'aide que pourrait apporter le tourisme à l'entretien des vestiges historiques de la Qal'a afin d'éviter leur décrépitude et pourquoi pas même contribué au financement des reprises des fouilles qui se sont arrêtées depuis plus d'une trentaine d'années. L'enjeu étant bien malheureusement non renouvelable, l'urgence de la prise de conscience commence à se ressentir chez les groupes de sauvegarde, reste de trouver les mécanismes adéquats pour tirer la sonnette d'alarme des législateurs et de là les gestionnaires du patrimoine archéologique. 


\section{Bibliographie}

AL MUQADDASI, 1950, Description de l'occident musulman au IV e = Xe siècle, traduction Ch. Pellat, Paris.

ASSAM-BALOUL Nadia, 2013, Le rôle des acteurs locaux dans le développement muséal Entre motivations culturelles et enjeux économiques. Exemple du musée régional de Tizi-Ouzou. Université Mouloud Mammeri, département Architecture Tizi-Ouzou Algérie) 50ème colloque de l'ASRDLF 8 -11 juillet 2013 Mons, Belgique. AUTISSIER Anne-Marie, 2008, L'Europe des festivals. De Zagreb à Edinbourg points de vue croisées, éditions de l'attribut, Toulouse.

BLAIS Mireille \& MARTINEAU Stéphane, 2006, "L'analyse inductive générale : description d'une démarche visant à donner un sens à des données brutes». Les recherches qualitatives, vol. 26, no 2, p. 1-18.

BENITO Luc in France FESTIVALS (2003) La musique a-t-elle besoin des festivals ?, Actes du colloque du 13-14 novembre 2003, à l'Abbaye de Royaumont, Val-d'Oise, Ed. La Scène-Magazine des professionnels du spectacle, Paris, p.5

BENSEDDIK-SOUKI \& CHAABI, (sous la dir. de), 2012, la valorisation des monuments historiques en Algérie. Le cas du palais de l'Agha a Ferdjioua, mémoire de Magister, département d'architecture et d'urbanisme, faculté des sciences de la terre, de géographie et de l'aménagement du territoire, université Mentouri, Constantine, Algérie.

BLANCHET P, 1899, "La Kalaa des Beni-ammâd", Recueil de la Société Archéologique de la Province de Constantine.

BOUKHELIFA S, 2007, " Le tourisme algérien : Chronique d'une déliquescence annoncée », in http:/www.algeriantourism.com

BOUROUIBA Rachid, 1964, Rapport préliminaire de sur la compagne de fouilles de septembre 1964 à la Qal'a des bani Hammad, t. I

BOUROUIBA Rachid, 1971, Musées d'Algérie, SNED, Collection Art et Culture, Alger.

BOUROUIBA Rachid, 1975, La Qal'a des Bani Hammad, Minister de l'information et de la culture, Sous direction des beaux arts et Antiquités, Alger, p.94

CAZES, Georges et POTIER Françoise, 1996, Le tourisme urbain, Presses universitaires de France, Paris.

DAILLY, TORRENTE \& BARTHES (sous la dir. de), 2009, En quoi la mise en tourisme d'un patrimoine archéologique peut être un facteur de développement et de sensibilisation pédagogique durable ? Le cas de la citerne El-Nabih, mémoire de master 2 Tourisme et développement, université de Toulouse II- le Mirail. DIAMANTAKI Grayfallia \& TIARD Michel (dir), 2010, Les festivals : moteurs de la valorisation du patrimoine et de l'attractivité touristique d'un territoire. «Le Festival 
de la Photographie Les Rencontres d'Arles et la ville d'Arles». Mémoire de Master II professionnel Tourisme. Valorisation touristique des sites culturels. Université de paris 1 - panthéon Sorbonne institut de recherche et d'études supérieures du tourisme

DE BEYLIE J, 1909, La Kalaa des Beni-Hammâd, une capitale berbère de l'Afrique du Nord au $\mathrm{XI}^{\mathrm{e}}$ siècle, Leroux, Paris.

DECROP Alain, 2004, «Trustworthiness in Qualitative Tourism Research ». Dans Qualitative Research in Tourism Ontologies, Epistemologies and Methologies. Sous la dir. de Jenny Phillimore et Lisa Goodson. Routledge, New York, p. 156-169. DIRECTIONDE LAPLANIFICATIONET DE L'AMENAGEMENT DU TERRITOIRE, 2014, annuaire statistique de la wilaya de M'sila.

EL BEKRI, 1859, El Bekri écrivait au onzième siècle, Traduction de Slane. Journal asiatique, p. 11.

EL-IDRISI, 1866, description de l'Afrique et de l'Espagne, traduction, tard de Dozy et de Goeje, Leiden, Paris.

FLECHET Anaïs, GOETSCHEL Pascale, HIDIROGLOU Patricia, JACOTOT Sophie, GRAVARI-BARBAS Maria \& JACQUOT Sébastien, 2008, Impacts socio-économiques de l'inscription du site sur la liste du patrimoine mondial ; une revue de la littérature, rapport à l'UNESCO, Centre du Patrimoine Mondial.

GRAVARI-BARBAS Maria \& JACQUOT Sébastien, 2010, Villes françaises du patrimoine mondial et tourisme ; Protection, gestion, valorisation, Actes de la journée organisée par la chaire UNESCO «culture, Tourisme Développement » L'IREST et L'EIREST, Université Paris1 Panthéon-Sorbonne, la Convention FranceUnesco, ICOMOS-France.

GOLVIN Lucien, 1965, Recherches archéologiques de la Kalaa des Béni Hammad, Maisonneuve et Larose, Paris.

GOLVIN Lucien, 2000, " Hammadides », Encyclopédie berbère, 22 | Hadrumetum - Hidjaba, Edisud, Aix-en-Provence, p. 3334-3345

HARRISON David \& HITCHCOCK Michael, 2005, The politics of World Heritage : Negotiating Tourism and Conservation, Clevedon : Channel View Publications.

HOOG Emmanuel, 1991, « La France en festivals », entretien avec le directeur du Festival de poésie du Haut Allier, et fonctionnaire au ministère de la culture, Le Monde, édition du 30 septembre 1991, p. 15

IBN KHALDOUN, 1852, Histoire des Berbères et des dynasties musulmanes de l'Afrique Septentrionale, traduction Baron de Slane, imprimerie du gouvernement, Alger

IDIR Mohamed Sofiane \& FERGUENNE Ameziane (dir), 2013, Valorisation du patrimoine, tourisme et développement territorial en Algérie : Cas des régions de Béjaia et de Djanet dans le Tassili N'ajjer, Thèse de doctorat en sciences économiques, 
université de Grenoble, France.

JUDD. D \& FAINSTEIN, S. (sous la dir. de), 1999, The Tourist City, Yale University Press, New Haven,

LAW CHRISTOPHER M., 1993, Urban Tourism, Attracting Visitors to Large Cities, , Mansell, Londres.

LAZAROTI Olivier, 2003, Tourisme et patrimoine ; ad augusta per angustia/Tourism and Heritage : ad augusta per angustia, Annales de géographie, volume $12 n^{\circ} 629$, p.91-110

LEBAAL Noureddine \& ZEROUALA Med Salah, 1997, sauvegarde du patrimoine architectural dans les pays en voie de développement, cas de l'Algérie, actes du séminaire international en architecture,L'Architectureet la ville dans le contexte algérien, stratégies conceptuelles appropriées, Biskra le 10 et 11 Novembre, pp.281289

MARCAIS GeORges, 1908, "La Kalaa des Beni-Hammâd”, Recueil des Notices et Mémoires de la Société Archéologique du département de Constantin et. XLII, p. 161-188.

MERCIER Sophie \& BOUCHARD Diane, 2014, Tourisme culturel et festivals Opportunités et limites d'un tel partenariat. Le Printemps de Bourges - Jazz in Marciac - Les Eurockeennes de Belfort.

MIRIEU de LABARRE Eric, 2004, « Plaidoyer pour un nouveau régime des abords », in CRIDEAU-CNRS, Les monuments historiques, un nouvel enjeu ? L'Harmattan, Volume 2, Paris, p. 11-62.

MOINE Caroline \& VERLAINE Julie, dirs, Une histoire des festivals, XXe-XXIe siècle, Publications de la Sorbonne, coll. Histoire contemporaine, Paris, 2013, 354 pages.

Pascal Ory (2013) «Qu'est-ce qu'un festival ? Une réponse par l'histoire » in Une histoire des festivals, XXe-XXIe siècle, Paris, Publications de la Sorbonne, coll. Histoire contemporaine, pp 19-32

NEGRIER Emmanuel \& JOURDA Marie Thérèse, 2007, les nouveaux térritoires des festivals, France Festivals-Michel de Maule, Paris.

PAGE Stephen, 1995, Urban Tourism, Routledge, London.

PEARCE Douglas, 1987, Tourism Today: A Geographical Analysis, Longman, Harlow.

ROY Simon N, 2009, «L'étude de cas». Dans Recherche sociale : de la problématique à collecte des données, sous la dir. de Benoit Gauthier, Se édition. Presses de 1 ‘Université du Québec, Québec, p. 199-22S.

SALADIN H, 1909, La kalaa des Beni-Hammad [Général L. de Beylié. La Kalaa des Beni-Hammad, une capitale berbère de l'Afrique du Nord au XIe siècle]. In: Journal des savants. 7 année, Juin 1909. pp. 255-260 
SCOTT Noel \& JAFARI Jafar, 2010 Bridging Tourism Theory and Practive, Tourism in the Muslim World, Emerald Group Publishing Limited, United Kingdom SMAHI, BOUMRAR \& DAHMANI (dir), 2013, les festivals locaux, outils de valorisation du patrimoine. Cas de la wilaya de Tizi Ouzou, mémoire de Master II option développement local, Tourisme et Valorisation du territoire, Faculté des Sciences économiques, commerciales et de Gestion, université Mouloud Maamri de Tizi Ouzou.

VELLE \& CLAVERIE (sous la dir. de), 2014, Festivals et développement des territoires : Le cas du Festival International de Théâtre de Rue d'Aurillac, Mémoire de Master, institut supérieur du tourisme, de l'hôtellerie et de l'alimentation, université de Toulouse II- le Mirail 